\title{
Análise da prevalência de acidente vascular encefálico em pacientes assistidos por uma instituição hospitalar
}

Analysis of the prevalence of stroke in patients assisted by a hospital Análisis de la prevalencia de accidente vascular encefálico en pacientes asistidos por un hospital

\author{
Patrick Leonardo Nogueira da SILVA ${ }^{(1)}$ \\ Luciana Soares dos SANTOS ${ }^{(2)}$ \\ Débora Danielly Dias RIBEIRO ${ }^{(3)}$ \\ Hellem Daiany Gonçalves GUIMARÃES ${ }^{(4)}$ \\ Kênia Alencar Froes ESTEVES ${ }^{(2)}$ \\ Elaine Cristina Santos ALVES ${ }^{(2)}$ \\ Hellen Veloso Rocha MARINHO ${ }^{(2)}$
}

Recebido: 20 out 2016

Revisado: 11 jul 2017

Aceito: 22 nov 2017

\section{Autor de}

correspondência:

Patrick Leonardo

Nogueira da Silva

patrick_mocesp70@hotmail .com

Conflito de interesses:

Os autores declaram não

haver nenhum interesse profissional ou pessoal que possa gerar conflito de interesses em relação a este manuscrito.

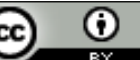

\footnotetext{
(1) Prefeitura Municipal de Espinosa, Espinosa, MG, Brasil.

(2) Universidade Estadual de Montes Claros - Unimontes, Montes Claros, MG, Brasil.

(3) Prefeitura Municipal de Francisco Sá, Francisco Sá, MG, Brasil.

(4) Faculdades Integradas Pitágoras - FIP-MOC, Montes Claros, MG, Brasil.
}

\section{Resumo}

O acidente vascular encefálico é uma das maiores causas de morbimortalidade em todo o mundo. Caracteriza-se por déficit neurológico em uma área encefálica secundária à lesão vascular, e representa um grupo de doenças com manifestações clínicas semelhantes. Este estudo objetivou analisar a prevalência do acidente vascular encefálico em pacientes assistidos por uma instituição hospitalar. Trata-se de um estudo descritivo na qual sua amostra compreendeu 288 prontuários de pacientes com diagnóstico de acidente vascular encefálico internado no período de janeiro a dezembro de 2008. Utilizou-se um formulário como instrumento de coleta de dados. O tratamento dos mesmos se deu por meio de epidemiologia descritiva. Houve prevalência do sexo masculino, entre 70-79 anos e pardos. Apresenta como um dos principais fatores de risco a hipertensão arterial sistêmica $(62,8 \%)$ e que leva a diversas manifestações clínicas e déficits neurológicos, em destaque, a hemiplegia (39,2\%). Quanto ao tipo de episódio, $65 \%$ não foram especificadas de forma a apontar o tipo isquêmico como o mais frequente (72\%). Conclui-se que o acidente vascular encefálico isquêmico mostrou-se mais prevalente e recorrente em idosos do sexo masculino na qual a hipertensão arterial sistêmica foi o principal fator de risco causador da doença.

Descritores: Acupuntura; Ansiedade; Estresse Psicológico; Neoplasias da Mama; Revisão. 


\begin{abstract}
Stroke is a major cause of morbidity and mortality worldwide. Characterized by a secondary neurological deficit in brain area to vascular injury, and is a group of diseases with similar clinical manifestations. This study aimed to analyze the prevalence of stroke in patients assisted by a hospital. This is a descriptive study in which a sample consisted of 288 records of patients diagnosed with stroke admitted to the period from January to December of 2008. We used a form as a collection tool data. Treatment of these occurred by descriptive epidemiology. There was prevalence in males, between 70-79 years and browns. It presents one of the main risk factors systemic arterial hypertension (62.8\%) and that leads to various clinical manifestations and neurological deficits, highlighted, hemiplegia (39.2\%). Regarding the type of episode, $65 \%$ were not specified to point the ischemic stroke as the most common (72\%). It was concluded that ischemic cerebrovascular accident was more prevalent and recurrent in the elderly male patients in whom systemic arterial hypertension was the main risk factor causing the disease.
\end{abstract}

Keywords: Acidente Vascular Encefálico; Fatores de Risco; Sinais e Sintomas; Manifestações Neurológicas; Perfil de Saúde.

\begin{abstract}
Resumen
El accidente cerebrovascular es una causa importante de morbilidad y mortalidad en todo el mundo. Caracterizado por un déficit neurológico secundario en la zona del cerebro a la lesión vascular, y es un grupo de enfermedades con manifestaciones clínicas similares. Esto estudio objetivó analizar la prevalencia de ictus en pacientes con la asistencia de un hospital. Este es un estudio descriptivo en el que la muestra incluyó 288 historias clínicas de pacientes con diagnóstico de accidente cerebrovascular ingresados en el período de enero a diciembre de 2008. Se utilizó un formulario como una herramienta de recolección datos. El tratamiento de ellos fue dado por la epidemiología descriptiva. Hubo prevalencia en los hombres, entre 70-79 años y marrones. Presenta uno de los principales factores de riesgo La hipertensión (62,8\%) y que conduce a diversas manifestaciones clínicas y los déficits neurológicos, destacó, hemiplejía (39,2\%). Respecto al tipo de episodio, el $65 \%$ no se especifica para señalar el tipo isquémico como la más frecuente (72\%). Se concluye que el accidente cerebrovascular isquémico es más frecuente y recurrente en hombres de edad avanzada en la que la hipertensión es la causa principal de la enfermedad factor de riesgo.
\end{abstract}

Palabras-claves: Acupuntura; Ansiedad; Estrés Psicológico; Neoplasias de la Mama; Revisión.

\title{
Introdução
}

O acidente vascular encefálico - AVE pode ser definido como um déficit neurológico (transitório ou definitivo) em uma área encefálica secundária à lesão vascular, e representa um grupo de doenças com manifestações clínicas semelhantes, mas que possuem as seguintes subdivisões: hemorrágico, na qual envolve a hemorragia subaracnóidea, em geral decorrente da ruptura de aneurismas saculares congênitos localizados nas artérias do polígono de Willis e a hemorragia intraparenquimatosa, cujo mecanismo causal básico é a degeneração hialina de artérias intraparenquimatosas cerebrais; já o tipo isquêmico 
apresenta-se como o déficit neurológico resultante da insuficiência encefálica de suprimento sanguíneo, podendo ser temporário (episódio isquêmico transitório) ou permanente.

O AVE é uma das maiores causas de morbimortalidade em todo o mundo. ${ }^{2}$ No Brasil, apesar do decaimento nas taxas de mortalidade, o AVE ainda é a principal causa de morte. ${ }^{3}$ A incidência desta doença dobra a cada década após os 55 anos de idade, ocupando posição de destaque entre a população idosa. A prevalência mundial na população geral é estimada em $0,5 \%$ a 0,7\%. ${ }^{4}$ Além de sua elevada incidência, o AVE tem sido o principal motivo de internações hospitalares e incapacidades, resultando em sérias consequências médicas e sociais, tais como: as sequelas de ordem física, de comunicação, funcionais, emocionais, entre outras. ${ }^{5}$

Existem alguns fatores de risco que são associados à ocorrência do AVE e podem ser classificados como modificáveis e não-modificáveis. Os modificáveis são: Hipertensão Arterial Sistêmica - HAS, Diabetes Mellitus - DM, Tabagismo, Fibrilação Arterial - FA, Doenças Cardiovasculares - DCV, Doença Hiperlipidêmica, sedentarismo, estenose carotídea assintomática e ataque isquêmico transitório - AIT. Já os fatores nãomodificáveis são: a idade, o sexo, a raça, a hereditariedade e a etnia. O etilismo, anticorpo antifosfolipídeo, homocisteína elevada, processo inflamatório e infecções também são considerados fatores de risco. ${ }^{6}$ Quanto ao processo de recuperação do paciente vítima de AVE, este estágio pode relacionar-se como tratamento precoce, local e extensão da lesão inicial, idade, plasticidade do sistema nervoso, incapacidade de atingir uma meta motora relacionada com o movimento funcional, estado pré-mórbido do paciente e motivação e atitude do paciente com relação à recuperação. ${ }^{7}$

A cerca das aquisições em pesquisa sobre esta temática, reforça-se que há uma enorme quantidade de recursos investidos em pesquisa no mundo todo, a fim de reduzir a morbimortalidade da doença. ${ }^{1}$ Parte destes estudos embasa-se em fatores que são o perfil epidemiológico, o tratamento de suas complicações, avaliação e prevenção. Contudo, a 
morbimortalidade pela doença vem crescendo pelo déficit no monitoramento dos fatores de risco. $^{8}$ Diante do exposto, objetivou-se analisar a prevalência do AVE em pacientes assistidos por uma instituição hospitalar.

\section{Metodologia}

Artigo da monografia intitulada "Perfil dos pacientes acometidos por acidente vascular cerebral no Hospital Santa Casa de Montes Claros" apresentada ao Departamento de Enfermagem da Universidade Estadual de Montes Claros/UNIMONTES. Montes Claros (MG), Brasil. 2009.

Trata-se de um estudo descritivo, realizado no Serviço de Arquivo Médico e Estatístico - SAME do Hospital Santa Casa de Misericórdia - HSCM da cidade de Montes Claros, Minas Gerais, Brasil. No SAME estão localizados os registros de internação de todos os pacientes do Hospital, sendo que neste setor é possível verificar todo o histórico dos pacientes, desde a sua entrada até a sua saída, na qual os óbitos estão inclusos.

Foi enviada uma carta de apresentação e um Termo de Consentimento Institucional - TCI à Direção Clínica da HSCM, para autorização do estudo. A instituição foi devidamente orientada quanto às diretrizes da pesquisa e a mesmas assinou o TCI de modo a autorizar a realização da pesquisa.

A coleta de dados foi realizada durante o período de julho a agosto de 2009. Foram utilizados critérios específicos de amostragem, pois todos os pacientes que compõem a população foram estudados conforme os critérios de inclusão. Foram incluídos nesta pesquisa todos os registros dos pacientes que internaram no HSCM de janeiro a dezembro de 2008 na qual evoluíram com AVE.

Foram analisados 288 prontuários de pacientes com diagnóstico de AVE. Foi realizado tratamento estatístico adequado observando a frequência absoluta e a frequência percentual dos dados coletados. Para a coleta de dados, usaram-se as 
informações registradas em prontuários de pacientes diagnosticados com AVE e assistidos nesta instituição. A amostra foi selecionada a partir do levantamento estatístico de internação da instituição no ano de 2008, por meio de um sistema de informação da própria instituição.

Foi utilizado um formulário de elaboração própria baseado nos dados epidemiológicos do prontuário clínico como instrumento de coleta de dados. As variáveis investigadas neste estudo foram: sexo, idade, raça/cor, fatores de risco, manifestações clínicas e déficits neurológicos, tipo de episódios de AVE e etiologia do AVE.

Após coleta dos dados, estes foram armazenados no banco de dados StatisticalPackage for the Social Sciences (SPSS $\left.{ }^{2}\right)$, versão 20.0 e tabulados a partir do Office Microsoft Exce/ $\mathbb{R}$, versão 13.0, expostos em gráficos e tabelas para posteriormente serem discutidos por meio de epidemiologia descritiva.

O estudo obedeceu aos preceitos éticos estabelecidos pela Resolução n 466/2012 do Conselho Nacional de Saúde - CNS na qual regulamenta a pesquisa envolvendo seres humanos. $^{9}$ O projeto de pesquisa foi apreciado e aprovado pelo Comitê de Ética em Pesquisa da Universidade Estadual de Montes Claros - CEP-UNIMONTES, sob o parecer consubstanciado $n^{\circ} 1.548 / 2009$.

\section{Resultados}

Dos 288 prontuários analisados, o AVE foi mais prevalente em pacientes do sexo masculino (53,1\%), com faixa etária entre 70-79 anos (24,6\%), e ausência de especificação da raça/cor na maior parte dos prontuários (56,3\%). Aos que especificaram, prevaleceu a cor parda (37,5\%) (Tabela 1). 
Tabela 1. Perfil socioeconômico de pacientes com diagnóstico de AVE assistidos em uma instituição hospitalar. Montes Claros, MG, 2009

\begin{tabular}{|c|c|c|}
\hline Variáveis & $n^{*}$ & $\%$ \\
\hline \multicolumn{3}{|l|}{ Sexo } \\
\hline Masculino & 153 & 53,1 \\
\hline Feminino & 135 & 46,9 \\
\hline \multicolumn{3}{|l|}{ Faixa etária (anos) } \\
\hline $10-19$ & 3 & 1 \\
\hline $20-29$ & 4 & 1,3 \\
\hline $30-39$ & 13 & 4,5 \\
\hline $40-49$ & 32 & 11,1 \\
\hline $50-59$ & 60 & 20,8 \\
\hline $60-69$ & 66 & 22,9 \\
\hline $70-79$ & 71 & 24,6 \\
\hline $80-89$ & 34 & 11,8 \\
\hline 90 ou + & 5 & 2 \\
\hline \multicolumn{3}{|l|}{ Raça/cor } \\
\hline Parda & 108 & 37,5 \\
\hline Branca & 18 & 6,2 \\
\hline Não especifica & 162 & 56,3 \\
\hline
\end{tabular}

Fonte: SAME

Nota: ${ }^{*} \mathrm{n}=288$

Da população estudada, os fatores de risco para o AVE mais prevalentes foram a HAS (62,8\%), seguida das DCV (25,3\%), e DM (13,5\%). Em 5,2\% dos prontuários foram encontradas outras causas para aquisição da doença, tais como a obesidade e o estresse. Em 13,8\% não houve especificação de nenhum fator de risco (Tabela 2).

Quanto às manifestações clínicas e déficits neurológicos decorrentes do AVE observados neste estudo, a maior parte dos pacientes cursou com hemiplegia $(39,2 \%)$, seguida da hemiparesia (35,8\%) e afasia (31,9\%). Em 5,9\% dos prontuários foram observadas outras manifestações clínicas de menor prevalência neste estudo, sendo estes: parestesia, aumento da pressão intracraniana - PIC e edema encefálico (Tabela 3). Assim como os fatores de risco, as manifestações clínicas também podem acometer a outros pacientes, sendo a mesma computada mais de uma vez. 
Tabela 2. Fatores de risco de pacientes com diagnóstico de AVE assistidos em uma instituição hospitalar. Montes Claros, 2009

\begin{tabular}{lcc}
\multicolumn{1}{c}{ Variáveis } & $\mathbf{n}^{*}$ & $\%$ \\
Fatores de risco & & \\
HAS & 181 & 62,8 \\
DCV & 73 & 25,3 \\
DM & 39 & 13,5 \\
Etilismo & 29 & 10 \\
Tabagismo & 27 & 9,3 \\
Dislipidemias & 5 & 1,7 \\
Outros & 15 & 5,2 \\
Não especificados & 40 & 13,8 \\
\hline
\end{tabular}

Fonte: SAME

Nota: ${ }^{*} \mathrm{n}=288$

Tabela 3. - Manifestações clínicas e déficits neurológicos de pacientes com diagnóstico de AVE assistidos em uma instituição hospitalar. Montes Claros, 2009

\section{Variáveis}

$n^{*}$

Manifestações clínicas e déficits neurológicos

Hemiplegia
Hemiparesia
Afasia
Disartria
Cefaleia intensa súbita
Confusão mental
Paresia
Disfagia
Paralisia facial
Alterações no nível de consciência
Náuseas e vômitos
Convulsões
Sonolência
Ataxia
Coma
Distúrbios visuais
Rigidez de nuca
Tonturas
Outras

Fonte: SAME

Nota: ${ }^{*} \mathrm{n}=288$
ISSN 2179-6750 
Em se tratando da recorrência do $A V E$, observou-se que 21,8\% foram episódios recorrentes e $12,8 \%$ foi o primeiro episódio. Observou-se que na maior parte dos prontuários analisados (65,4\%) essa informação não foi especificada pelos profissionais que elaboraram a anamnese do paciente assistido. $O$ perfil dos pacientes quanto à etiologia do $A V E$, demonstra maior prevalência quanto ao tipo isquêmico (72,5\%) comparado ao tipo hemorrágico (19\%). Em 8,5\% dos casos, essa classificação não foi especificada nos prontuários (Tabela 4).

Tabela 4. Classificação quanto ao tipo do episódio e do AVE de pacientes assistidos em uma instituição hospitalar. Montes Claros, 2009

\begin{tabular}{lcc}
\multicolumn{1}{c}{ Variáveis } & $\mathbf{n}^{*}$ & $\%$ \\
Tipo de episódio & & \\
$\quad$ Primeiro episódio & 37 & 12,8 \\
Episódio recorrente & 63 & 21,8 \\
Não especificado & 188 & 65,4 \\
Classificação do AVE & & \\
Isquêmico & 209 & 72,5 \\
Hemorrágico & 55 & 19 \\
Não especificado & 24 & 8,5 \\
\hline
\end{tabular}

Fonte: SAME

Nota: ${ }^{*} \mathrm{n}=288$

\section{Discussão}

Trata-se de uma patologia que tende a acometer mais homens, o que pode ser justificado pela maior preocupação dada pelas mulheres à sua saúde. Apresenta como principais fatores de risco a HAS e DCV, sendo o AVE isquêmico o de maior prevalência no estudo realizado. O AVE pode-se dar de modo rápido, e caso não seja tratado eficazmente 
pode evoluir para sequelas que acaba intervindo na qualidade de vida dos pacientes, comprometendo até mesmo a realização de suas atividades básicas diárias.

Verifica-se que o resultado desta pesquisa corrobora com a maioria dos estudos revisados, os quais demonstram um predomínio da doença no sexo masculino. Estudo levantou o perfil dos pacientes acometidos por AVE, assistidos na Clínica de Fisioterapia Neurológica da Universidade de Passo Fundo - CFN-UPF, em que 72,09\%, de um total de 41 pacientes, correspondeu ao sexo masculino. ${ }^{10}$ Tal fato pode estar relacionado com a diferença de atitude de homens e mulheres em relação à situação de saúde, já que as mulheres são mais atentas ao aparecimento de sintomas, procuram consultas médicas mais do que os homens, declaram mais suas doenças, consomem mais medicamentos e se submetem a mais exames, o que facilita o diagnóstico precoce e confere um melhor prognóstico, reduzindo a necessidades de internação e aumentando as chances de maior sobrevida. ${ }^{11}$

Pode-se inferir que os dados deste estudo corroboram com os resultados de outro estudo em que também encontraram maior prevalência numa média de idade de 52 anos e concentração dos casos da doença nas faixas etárias de 40 a 59 anos de idade..$^{5}$ aumento de episódios de doença nesta faixa etária se deve a uma maior expectativa de vida da população, bem como ao aumento na sobrevida dos pacientes com AVE e à melhoria no atendimento desses pacientes nos setores de alta e média complexidade. $\mathrm{O}$ aumento na prevalência do AVE na população idosa é esperado, visto que a idade é o principal fator de risco não-modificável, reforçando a necessidade de medidas urgentes de prevenção e controle de fatores de risco para doença cerebrovascular, a serem realizadas pela Atenção Primária, que promovam a redução dos comportamentos de risco na população adulta, com o intuito de permitir que atinja o envelhecimento de forma saudável. $^{12}$

Um estudo ${ }^{9}$ aponta que, com relação à raça dos indivíduos, 90,7\% da amostra era caracterizada por pacientes de raça branca, contrapondo os dados da literatura, os quais 
citam uma incidência de AVE duas vezes maior na raça negra, como pode ser visto no estudo. $^{13}$ A divergência do resultado de seu estudo, em relação à literatura, pode ser explicada pela predominância da raça branca no estado do Rio Grande do Sul, onde foi realizada a pesquisa. ${ }^{9}$ Quanto aos fatores de risco, os números observados seguem o mesmo padrão de estudo, ${ }^{1}$ no qual a HAS se portou como um fator de risco preditivo poderoso para o AVE, com frequência de 67,5\% para qualquer tipo de AVE. As DCV constituíram o segundo e mais importante fator de risco, com prevalência de $24,6 \%$.

Tais dados ainda se assemelham em outro estudo ${ }^{14}$ acerca do AVE, na qual 81,6\% pacientes avaliados apresentavam HAS; 57,9\% apresentou alguma DCV; 36,8\% já sofreram AVE prévio; $34,2 \%$ eram ou já foram tabagistas; $26,3 \%$ apresentavam DM; $37,9 \%$ eram dislipidêmicos; 2,6\% sofreu AIT prévio; 5,3\% não apresentavam fatores de risco; e 15,8\% apresentavam outros fatores, tais como: histórico familiar de AVE, etilismo, obesidade e lúpus eritematoso sistêmico - LES. Apesar dos dados desta pesquisa estar de acordo com os dados da literatura, estes podem ser discutíveis, uma vez que durante a avaliação dos prontuários, percebeu-se que várias anamneses não continham informações a respeito dos fatores de risco para AVE apresentados pelos pacientes, assim, esses prontuários foram contabilizados no estudo como não especificados. Ressalta-se que o mesmo fator de risco pode acometer vários pacientes de forma a ser contabilizado mais de uma vez no resultado.

Estes resultados relacionam-se com os dados encontrados em um estudo, ${ }^{15}$ cujas manifestações clínicas diagnosticadas após o AVE incluem fraqueza ou dormência numa parte do corpo, dificuldade em falar, compreender, ler ou escrever, piora súbita da visão, cefaleia súbita intensa e vômitos, visão dupla, desequilíbrio, vertigem e tonturas, convulsões, desmaios ou sonolência e rigidez de nuca. A maioria dos AVE surge de maneira insidiosa, apresentam evolução rápida e causam lesões cerebrais. O paciente pode piorar progressivamente durante algumas horas ou dias e, muitas vezes, apresentar sérios 
déficits neurológicos de ordem física, motora, de comunicação, funcionais, visuais e emocionais, reduzindo, assim, sua qualidade de vida. ${ }^{16}$

Houve deficiência de informações nesta variável deste estudo, no entanto, estes estão de acordo com os estudos realizados nos Estados Unidos da América - EUA na qual afirma que, nesse país, $20 \%$ dos episódios de AVE ao ano são recorrentes ${ }^{17}$ Ainda de acordo o mesmo autor, quanto ao risco de recorrência de AVE, situa-se entre 15 e $30 \%$ em cinco anos, o que corresponde a uma chance nove vezes maior que a da população geral.

O novo episódio da doença cerebrovascular geralmente tem maior gravidade para o paciente e implica em significativo ônus para o sistema de saúde. A demência, o declínio da função cognitiva e a incapacidade motora são complicações frequentes e temidas após a recorrência do AVE, com importantes implicações socioeconômicas. ${ }^{17}$

Em contrapartida, outro estudo, ${ }^{10}$ revelou que a maioria da população estudada (86\%) tinha sofrido AVE pela primeira vez, 11,6\% tiveram episódio recorrente em 2,4\% o prontuário não continha esta informação. Em se tratando da incidência de $A V E$, embora haja inexistência de parte das informações, os resultados desta pesquisa vão ao encontro com dados apresentados por outros estudos em que dos pacientes analisados, houve maior incidência do AVE isquêmico em detrimento ao AVE hemorrágico. ${ }^{12,10}$ Um número considerável de prontuários $(n=24 ; 8,5 \%)$ em que não foi especificado o tipo de AVE do paciente pode ser explicado pela não realização da Tomografia Computadorizada - TC de encéfalo ou Ressonância Magnética (RM) em alguns casos, ou ainda pelo fato do resultado desses exames não se apresentarem bem esclarecidos. Conforme o protocolo institucional para solicitação e realização de TC e RM, os mesmos somente são solicitados em caso de internação do paciente pelo médico, via pronto socorro/ambulatório, com permanência mínima obrigatória ou para pacientes já internados na qual o exame é requerido durante o período da internação. Sendo assim, em decorrência da clínica estável do paciente, o exame não é solicitado devido o paciente não apresentar justa causa para internação. 
Para o diagnóstico do $A V E$, é necessário não só uma avaliação minuciosa dos fatores de risco de DCV e exame físico, mas é também indispensável a realização de exames complementares através do imageamento encefálico por TC e/ou RM, já que, somente através destes, é possível caracterizar de forma segura o AVE em isquêmico ou hemorrágico, bem como identificar a sua etiologia e intervir rapidamente. ${ }^{18} \mathrm{O}$ número considerável de prontuários na qual não foi especificado o tipo de AVE pode ser explicado pela não realização da TC de encéfalo ou RM, ou ainda pelo fato do resultado desses exames não se apresentarem bem esclarecidos.

Neste contexto, observa-se o fato de que, mesmo em se tratando de uma patologia tão relevante, foi possível perceber um déficit no preenchimento de dados pela instituição pesquisada, o que muitas vezes compromete a avaliação por parte da mesma sobre a qualidade do atendimento prestado, e também sobre as medidas que podem ser tomadas para que cada vez mais se possa prestar uma assistência eficaz, e que possa ao menos minimizar as sequelas deixadas pelo AVE. A clareza dos dados devidamente registrados estipula estratégias viáveis que favoreçam a redução dos danos proporcionados pela doença. Sendo assim, sugerimos maior rigor no registro e avaliação desses dados que levam a ganhos tanto por parte da Instituição bem como pela população atendida por esta.

\section{Conclusão}

Este estudo permitiu inferir que o AVE configura-se como uma das maiores causas de morbimortalidade do cenário estudado. Conclui-se que o AVE isquêmico mostrou-se mais prevalente e recorrente em idosos do sexo masculino na qual a hipertensão arterial sistêmica foi o principal fator de risco causador da doença.

Portanto, faz-se necessário que a família e o profissional de saúde saibam identificar precocemente os sinais e sintomas do AVE a fim de intervir de forma eficaz, bem como amenizar os efeitos em longo prazo dos fatores de risco de forma a programar mudanças 
de hábitos de vida saudáveis e retardar o aparecimento desta doença propiciando o aumento na qualidade de vida do paciente.

\section{Referências}

1. Pedroso VSP, Souza LC, Teixeira AL. Síndromes neuropsiquiátricas associadas a acidentes vasculares encefálicos: revisão de literatura. J Bras Psiquiatr. 2014;63(2):165-76. http://dx.doi.org/10.1590/0047-2085000000021.

2. Feigin VL, Forouzanfar $M H$, Krishnamurthi $R$, Mensah GA, Connor M, Bennett DA, et al. Global and regional burden of stroke during 1990-2010: findings from the Global Burden of Disease Study 2010. Lancet. 2014 Jan 18;383(9913):245-54. https://doi.org/10.1016/S01406736(13)61953-4.

3. Carvalho MA, Coutinho APO, Carvalho GDA, Queiroz DTG, Santos SR. Epidemiologia dos acidentes vasculares encefálicos atendidos por meio do serviço de atendimento móvel de urgência. Rev Enferm UFPE on line [Internet]. 2015;9(3):1015-21. https://doi.org/10.5205/19818963-v9i3a10429p1015-1021-2015.

4. Costa TF, Costa KNFM, Martins KP, Fernandes MGM, Brito SS. Sobrecarga de cuidadores familiares de idosos com acidente vascular encefálico. Esc Anna Nery [Internet]. 2015 [acesso 15 fev. 2017];19(2):350-5. http://dx.doi.org/10.5935/1414-8145.20150048.

5. Mochizuki L, Bigongiari A, Franciulli PM, Francica JV, Alonso AC, Ervilha UF, et al. The effect of gait training and exercise programs on gait and balance in post-stroke patients. Med Express. 2015;2(4):1-5. http://dx.doi.org/10.5935/MedicalExpress.2015.04.01.

6. Costa VSP, Guimarães PSR, Fernandes KBP, Probst VS, Marquez AS, Fujisawa DS. Prevalence of risk factors for the occurrence of strokes in the elderly. Fisioter Mov. 2014 Oct-Dec;27(4):55563.http://dx.doi.org/10.1590/0103-5150.027.004.AO07.

7. Campos TF, Rodrigues CA, Farias IMA, Ribeiro TS, Melo LP. Comparação dos instrumentos de avaliação do sono, cognição e função no acidente vascular encefálico com a classificação internacional de funcionalidade, incapacidade e saúde (CIF). Rev Bras Fisioter [Internet]. 2012 [acesso 15 fev2017;16(1):23-9. Disponível em: http://www.scielo.br/pdf/rbfis/v16n1/05.pdf. 
8. Rodrigues JE, Sá MS, Alouche SR. Perfil dos pacientes acometidos por AVE tratados na clínica escola de fisioterapia da UMESP. Rev Neurociencias [Internet]. 2004 [acesso $15 \mathrm{fev}$ 2017];12(3):117-22. Disponível em:

https://www.revistaneurociencias.com.br/edicoes/2004/RN\%2012\%2003/Pages\%20from\%20RN \%2012\%2003.pdf.

9. Brasil. Ministério da Saúde. Conselho Nacional de Saúde. Resolução n 466, de 12 de dezembro de 2012. Aprova as diretrizes e normas regulamentadoras de pesquisas envolvendo seres humanos. Brasília, DF: CNS, 2012 [acesso 1 out 2014]. Disponível em: http://bvsms.saude.gov.br/bvs/saudelegis/cns/2013/res0466 1212 2012.html.

10. Mazzola D, Polese JC, Schuster RC, Oliveira SG. Perfil dos pacientes acometidos por acidente vascular encefálico assistidos na clínica de fisioterapia neurológica da Universidade de Passo Fundo. Rev Bras Prom Saude. 2007;20(1):22-7. http://dx.doi.org/10.5020/997.

11. Sales FM, Santos I. Perfil de idosos hospitalizados e nível de dependência de cuidados de enfermagem: identificação de necessidades. Texto Contexto Enferm. 2007;16(3):495-502. http://dx.doi.org/10.1590/S0104-07072007000300016.

12. Silva MF, Feitosa AR, Paz JA, Aikawa NE, Silva CA. Intracerebral hemorrhage with a favorable outcome in a patient with childhood primary angiitis of the central nervous system. Rev Bras Reumatol. 2016;56(4):366-70.http://dx.doi.org/10.1016/j.rbre.2014.05.007.

13. Nitrine R, Bacheschi LA. Neurologia que todo médico deve saber. São Paulo: Ateneu, 2003.

14. Yamashita LF, Fukujima MM, Granitoff N, Prado GF. Paciente com acidente vascular cerebral isquêmico já é atendido com mais rapidez no hospital São Paulo. Arq Neuropsiquiatr. 2004;62(1):96-102. http://dx.doi.org/10.1590/S0004-282X2004000100017.

15. Euzébio CJV, Rabinovich EP. Compreendendo o cuidador familiar do paciente com sequela de acidente vascular encefálico. Temas Psicol [Internet]. 2006 [acesso 15 fev 2017];14(1):63-79. http://pepsic.bvsalud.org/pdf/tp/v14n1/v14n1a08.pdf.

16. Sredni S, Sredni RAF. Cefaleia causada por dissecção de artéria vertebral: relato de caso. Rev Dor. 2012;13(4):382-4.http://dx.doi.org/10.1590/S1806-00132012000400014.

17. Piedade PR, Gagliardi RJ, Damiani IT, Nassar Junior AP, Fuzaro MM, Sanvito WL. Papel da curva de agregação plaquetária no controle da antiagregação na prevenção secundária do acidente vascular cerebral isquêmico. ArqNeuropsiquiatr. 2003;61(3):764-

7.http://dx.doi.org/10.1590/S0004-282X2003000500011. 
18. Pacheco FT, Littig IA, Gagliardi RJ, Rocha AJ. Multidetector computed tomography angiography in clinically suspected hyperacute ischemic stroke in the anterior circulation: an etiological workup in a cohort of Brazilian patients. ArqNeuropsiquiatr. 2015;73(5):40814.http://dx.doi.org/10.1590/0004-282X20150034

\section{Minicurrículo}

Patrick Leonardo Nogueira da Silva | ORCiD: 0000-0003-2399-9526

Especialista em Saúde da Família pela Universidade Estadual de Montes Claros - UNIMONTES. Enfermeiro da Prefeitura Municipal de Espinosa.

Luciana Soares dos Santos | ORCiD: 0000-0002-7413-7419

Graduação em Enfermagem pela Universidade Estadual de Montes Claros - UNIMONTES.

Débora Danielly Dias Ribeiro | ORCiD: 0000-0002-5853-2750

Especialista em Gestão dos Serviços e Sistemas de Saúde pela Universidade Estadual de Montes Claros (UNIMONTES). Enfermeira da Prefeitura Municipal de Francisco Sá.

Hellem Daiany Gonçalves Guimarães | ORCID: 0000-0002-7642-6169

Graduação em Enfermagem pelas Faculdades Integradas Pitágoras de Montes Claros - FIPMoc.

Kênia Alencar Froes Esteves | ORCID: 0000-0001-7026-8799

Mestre em Enfermagem pela Universidade Federal de São Paulo - UNIFESP. Professora do Departamento de Enfermagem da Universidade Estadual de Montes Claros - UNIMONTES.

Elaine Cristina Santos Alves | ORCID: 0000-0003-1357-9341

Mestre em Ciências da Saúde pela Universidade Federal de São Paulo - UNIFESP. Professora do Departamento de Enfermagem da Universidade Estadual de Montes Claros - UNIMONTES.

Hellen Veloso Rocha Marinho | ORCID: 0000-0002-3851-7270

Doutora em Ciências da Reabilitação pela Universidade Federal de Minas Gerais - UFMG. Professora do Programa de Pós-Graduação em Ciências da Saúde da Universidade Estadual de Montes Claros UNIMONTES. 